\title{
Fatores de risco cardiovascular e características sociodemográficas em idosos cadastrados em uma Unidade de Saúde da Família
}

\author{
Inaê Mariane de Jesus Bispo*, \\ Patrícia Honório Silva Santos* \\ Maria Aldenice Oliveira Carneiro* \\ Tamiles Daiane Borges Santana* \\ Marcos Henrique Fernandes* \\ Cesar Augusto Casotti* \\ Isleide Santana Cardoso Santos* \\ José Ailton Oliveira Carneiro*
}

\section{Resumo}

O envelhecimento populacional é acompanhado por modificações no padrão de morbimortalidade, resultando em maior exposição dos indivíduos aos fatores de riscos e agravos crônicos não transmissíveis. O objetivo do estudo foi identificar a prevalência de fatores de risco cardiovascular modificáveis e associar com as características sociodemográficas de idosos cadastrados em uma Unidade de Saúde da Família. Trata-se de um estudo transversal, realizado com 139 idosos com idade $\geq 60$ anos. A coleta de dados foi realizada por meio de um questionário estruturado, contendo questões referentes a informações sociodemográficas e fatores de risco cardiovascular (tabagismo, alcoolismo, hipertensão, diabetes, sedentarismo e sobrepeso/obesidade). Foram ainda realizadas medidas antropométricas como massa corporal, estatura e circunferência abdominal, o índice de massa corporal também foi calculado. Observou-se que dos 139 idosos recrutados, 75,5\% eram do sexo feminino. A idade média dos idosos foi 72,32 $\pm 8,4$ anos. As prevalências dos fatores de risco cardiovascular foram: hipertensão $64,7 \%$, obesidade $63,1 \%$, sedentarismo 46,8\%, circunferência abdominal elevada $41,3 \%$, diabetes mellitus 15,1\%, alcoolismo 13,8\% e tabagismo 10,1\%. Foi verificada associação do sedentarismo com grupo etário de 60-69 anos ( $p=0,004)$ e com cor/raça não branca ( $p=0,013)$; da circunferência abdominal elevada $(p=0,005)$ e do tabagismo $(p=0,03)$ com o sexo feminino; do consumo de álcool com o sexo masculino $(p=0,004)$ e da diabetes mellitus com classe econômica A e B $(p=0,04)$. Esse estudo evidenciou uma elevada prevalência de fatores de risco cardiovascular em idosos cadastrados em uma Unidade de Saúde da Família.

Palavras-chave: Idoso. Doenças cardiovasculares. Fatores de risco.

\begin{abstract}
Population aging is accompanied by changes in the pattern of morbidity and mortality, resulting in increased exposure of individuals to the risk factors and noncommunicable chronic diseases. This study aimed to identify the prevalence of modifiable cardiovascular risk factors and to associate with the sociodemographic characteristics of elderly registered in a Family Health Unit. This is an observational cross-sectional study, conducted with 139 elderly people. Data collection was carried out through a structured questionnaire containing questions regarding sociodemographic information and cardiovascular risk factors (smoking, alcoholism, hypertension, diabetes, sedentary and overweight/obesity). Anthropometric measurements also were realized, such as weight, height and abdominal circumference, the body mass index also was calculated. It was observed that of the 139 recruited elderly, $75.5 \%$ were female. The mean age of the elderly was $72.32 \pm 8.4$ years. The prevalence of cardiovascular risk factors were: hypertension $64.7 \%$; obesity $63.1 \%$; sedentary lifestyle 46.8\%; abdominal circumference high $41.3 \%$, diabetes mellitus $15.1 \%$; alcoholism $13.8 \%$ and smoking $10.1 \%$. Association was verified between sedentary and age group of $60-69$ years $(\mathrm{p}=0.004)$ and non-caucasians/race $(p=0.013)$; high abdominal circumference $(p=0.005)$ and smoking $(p=0.03)$ with female sex; consumption of alcohol with males $(p=0.004)$ and diabetes mellitus with economic class $A$ and $B(p=0.04)$. This study showed a high prevalence of cardiovascular risk factors in elderly registered in a Family Health Unit.
\end{abstract}

Keywords: Elderly. Cardiovascular disease. Risk factors.

DOI: 10.15343/0104-7809.20164003334342

\footnotetext{
*Universidade Estadual do Sudoeste da Bahia- UESB. Jequié, BA, Brasil.

** Universidade Estadual do Sudoeste da Bahia- UESB. Jequié, BA, Brasil. E-mail: patyhonorios@hotmail.com

Os autores declaram não haver conflitos de interesse.
} 


\section{INTRODUÇÃO}

O envelhecimento populacional é acompanhado por modificações no padrão de morbimortalidade, tendo como resultado maior exposição dos indivíduos aos fatores de riscos e agravos crônicos não transmissíveis, principalmente às doenças cardiovasculares ${ }^{1}$.

Segundo $\mathrm{WHO}^{2}$, as doenças cardiovasculares representam a principal causa de morte no mundo, com mais de três quarto dessas mortes ocorrendo em países com baixa e média renda. No Brasil, de acordo com dados provenientes do Departamento de Informática do SUS (DATASUS), as doenças do sistema circulatório acompanham a tendência mundial, visto que representam as mais relevantes causas de mortes do país, responsabilizando-se por $28,06 \%$ dos óbitos ocorridos no ano de $2013^{3}$.

Além do alto impacto sobre a mortalidade, as doenças cardiovasculares também contribuem para o aumento da morbidade, sendo que no ano de 2014 esteve na posição de terceira principal causa de internação, totalizando 1.133.235 internações no Brasil, o que representou cerca de $10 \%$ da morbidade hospitalar no período 3 . Neste sentido, essas doenças apresentam altos custos para os serviços de saúde, sendo responsáveis por um grande número de morte e invalidez, além de trazer transtornos para os familiares ${ }^{4}$.

Sendo assim, torna-se importante a identificação e controle dos fatores de risco cardiovascular para que estratégias de promoção da saúde sejam adotadas, além do diagnóstico e tratamento precoces ${ }^{5}$. Os fatores de risco cardiovascular podem ser classificados em não modificáveis, como: idade, sexo e hereditariedade e em modificáveis, como: obesidade, dislipidemias, diabetes, sedentarismo, tabagismo, hipertensão arterial, estresse e dieta inadequada ${ }^{6-8}$. Estes últimos estão relacionados aos aspectos comportamentais e ao estilo de vida, o que tornam as mudanças muito difíceis, exigindo que os indivíduos compreendam sua situação de saúde e necessidade de alterar seu modo de viver'.

O conhecimento da prevalência de cada fator de risco, isoladamente, fornece uma visão limitada sobre o problema, visto que esses fatores frequentemente ocorrem de maneira conjunta $^{9}$, e mesmo ocorrendo de maneira isolada acentua o risco de doença e morte ${ }^{10}$, de modo que quanto maior a simultaneidade de fatores de riscos presentes em indivíduos e populações, maior será a morbimortalidade das doenças cardiovasculares ${ }^{5}$.

Nesse sentido, torna-se fundamental a identificação da prevalência dos fatores de risco cardiovascular modificáveis em idosos, além de observar se possíveis características sociodemográficas contribuem para o desencadeamento dos mesmos, sabendo que muitos desses fatores são passíveis de alterações por meio de mudanças no estilo de vida, o que possibilita que estratégias de prevenção sejam adotadas. Diante do exposto, este estudo teve como objetivo identificar a prevalência de fatores de risco cardiovascular modificáveis e associar com as características sociodemográficas de idosos cadastrados em uma Unidade de Saúde da Família.

\section{METODOLOGIA}

Trata-se de um estudo observacional de corte transversal, baseado em dados provenientes do banco de dados da pesquisa intitulada "PETSaúde Jequié: Identificando o processo saúdedoença em população adstrita às Unidades de Saúde da Família", aprovada pelo Parecer no 084/2011 (CAAE 0064.0.454.000-11), do Comitê de Ética em Pesquisa da Universidade Estadual do Sudoeste da Bahia. Teve como local do estudo o domicílio dos idosos residentes no bairro Pau Ferro, do município de Jequié/BA, os quais são cadastrados na Unidade de Saúde da Família (USF) Giserlando Biondi.

A amostra foi realizada por conveniência com 139 idosos cadastrados na Equipe I da referida USF, sendo os critérios de inclusão ter idade igual ou superior a 60 anos, ser capaz de responder ao questionário e de realizar as medidas antropométricas, sendo excluídos da pesquisa os idosos que não se enquadraram nesses critérios e aqueles que não foram localizados em seus domicílios após três tentativas em dias e horários alternados. As diretrizes da resolução $n^{\circ}$ 466, de 12 de 
dezembro de $2012^{11}$ foram respeitadas e todos os participantes foram informados sobre os objetivos do estudo, procedimentos adotados e garantia de anonimato e sigilo das informações. A participação dos mesmos foi voluntária, não tendo benefícios econômicos. Após esclarecimentos do estudo, os idosos assinaram o termo de consentimento livre e esclarecido, concordando em participar da pesquisa.

A coleta de dados foi realizada no período de maio a novembro de 2013, por doze monitores de distintas áreas da saúde, previamente treinados, sob supervisão de seis preceptores e um tutor do Programa de Educação pelo Trabalho para Saúde/PET-Saúde (Grupo Idoso I), da Universidade Estadual do Sudoeste da Bahia, por meio de um questionário estruturado, contendo questões referentes às informações sociodemográficas e aos fatores de risco cardiovascular. Foram ainda realizadas medidas antropométricas como massa corporal, estatura e circunferência abdominal (CA), também foi calculado o índice de massa corporal (IMC).

\section{Fatores de Risco Cardiovascular (Variáveis dependentes)}

Os fatores de risco analisados nesse estudo foram: Tabagismo (avaliado por meio do autorrelato de fumo atual ou nos últimos 12 meses); Alcoolismo (avaliado pelo autorrelato de consumo atual ou nos últimos 12 meses); Hipertensão e Diabetes (autorreferida com base no diagnóstico médico); Insuficientemente ativo/sedentarismo (indivíduos que realizaram menos de 150 minutos por semana de atividades físicas moderadas e/ou vigorosas, avaliada por meio do International Physical Activity Questionnaire (IPAQ), um instrumento que quantifica por meio do autorrelato o tempo gasto em atividade física leve, moderada e vigorosa durante uma semana habitual, na versão curta $^{12}$ e adaptado para idosos no Brasil $^{13}$; Circunferência abdominal elevada substancialmente (homem $\geq 102 \mathrm{~cm}$ e mulher $\geq$ $88 \mathrm{~cm}^{14}$ avaliada na altura da cicatriz umbilical, utilizando uma trena antropométrica da marca Sanny, flexível e inextensível de 2 metros de comprimento, com precisão de uma casa decimal); e Sobrepeso/obesidade (IMC > 27
$\mathrm{Kg} / \mathrm{m} 2$, calculado a partir dos valores da massa corporal $(\mathrm{MC})$ e estatura (Est.): IMC $=\mathrm{MC}(\mathrm{Kg})$ / Est. $(\mathrm{m} 2))^{15}$.

Para a mensuração da MC foi utilizada uma balança digital portátil (Zhongshan Camry Eletronic, G-Tech Glass 6, China), onde o idoso foi orientado a ficar descalço, vestindo o mínimo de roupa possível.

Para medir a estatura, também permaneceu descalço na posição ereta, com pés juntos e com os calcanhares, nádegas e cabeça em contato com a parede e os olhos fixos num eixo horizontal paralelo ao chão (Linha de Frankfurt). Para realizar a medida correspondente à estatura, era colocado um esquadro sobre o topo da cabeça (vértex) do examinado, formando um ângulo de $90^{\circ}$ com a parede e marcando-se esse ponto, ao final de uma inspiração.

\section{Características Sociodemográficas (Variáveis independentes)}

As variáveis sociodemográficas analisadas foram: Sexo (masculino e feminino); Grupo etário (60 - 69, 70 - 79 e $\geq 80$ anos); Arranjo familiar (acompanhado e sozinho); Escolaridade (Até ensino médio incompleto e ensino médio completo ou mais); Classe econômica (classe A, de 35 a 46 pontos; classe B, de 23 a 34 pontos; classe $\mathrm{C}$, de 14 a 22 pontos; classe $\mathrm{D}$, de 8 a 13 pontos, e classe E, de 0 a 7 pontos) de acordo com a Associação Brasileira de Empresas de Pesquisa, que leva em consideração o grau de instrução do chefe da família e a presença de determinados bens de consumo em $\operatorname{casa}^{16} \mathrm{e}$ Cor/raça (branca e não branca).

\section{Análise estatística}

Inicialmente foi realizada uma análise descritiva por meio da frequência absoluta e relativa, média, desvio-padrão e amplitude. Para análise de associação entre cada fator de risco cardiovascular e as características sociodemográficas foram utilizados os testes de qui-quadrado de Pearson ou exato de Fisher, com um nível de significância de 5\% ( $p<0,05)$. Para análise dos dados utilizou-se o programa estatístico SPSS $®$, versão 21.0. 


\section{RESULTADOS}

Foram recrutados 139 idosos, sendo 105 $(75,5 \%)$ mulheres e $34(24,5 \%)$ homens. A idade dos idosos variou de 60 a 101 anos, com idade média de 72,32 \pm 8,4 anos. Quanto à etnia, os não brancos representaram a maior parte da amostra $(85,6 \%)$. No que se refere ao grau de instrução do chefe da família, $73,1 \%$ afirmaram ser analfabetos ou com ensino médio incompleto e quanto a classe econômica, $79(57,2 \%)$ se enquadraram nas classes econômicas D e E.

A tabela 1 mostra os fatores de risco para as doenças cardiovasculares, em que observase uma alta prevalência de idosos hipertensos $(64,7 \%)$ e obesos $(63,1 \%)$.
A Tabela 2 apresenta a associação dos fatores de risco modificáveis para doenças cardiovasculares com as variáveis sociodemográficas. Verificou-se associação do sedentarismo com grupo etário de 60-69 anos $(p=0,004)$ e com cor/raça não branca $(p=0,013)$.

O sexo feminino foi associado à circunferência abdominal elevada $(p=0,005)$ e ao tabagismo $(p=0,03)$, enquanto o sexo masculino foi associado ao maior consumo de álcool ( $p=0,004)$. O diabetes mellitus apresentou associação com classe econômica $(p=0,04)$. As demais associações podem ser visualizadas na Tabela 2.

Tabela 1 - Prevalência de fatores de risco modificáveis para doenças cardiovasculares em idosos adscritos a uma Unidade de Saúde da Família da cidade de Jequié, Bahia, Brasil, 2013.

\begin{tabular}{lll}
\hline \multicolumn{1}{c}{ Fatores de risco modificáveis } & N & $\%$ \\
\hline Sobrepeso/obesidade & 84 & 63,1 \\
Circunferência abdominal elevada & 57 & 41,3 \\
Insuficientemente ativo/sedentário & 65 & 46,8 \\
Tabagismo & 14 & 10,1 \\
Alcoolismo & 19 & 13,8 \\
Hipertensão arterial & 90 & 64,7 \\
Diabetes mellitus & 21 & 15,1 \\
\hline
\end{tabular}

Tabela 2 - Associação dos fatores de risco cardiovascular modificáveis em relação às características sociodemográficas de idosos adscritos a uma Unidade de Saúde da Família da cidade de Jequié, Bahia, Brasil, em 2013.

\begin{tabular}{lccccccc}
\hline $\begin{array}{c}\text { Grupo } \\
\text { etário }\end{array}$ & $\begin{array}{c}\text { Obesidade } \\
\mathbf{n}(\%)\end{array}$ & $\begin{array}{c}\text { Elevada } \\
\mathbf{n}(\%)\end{array}$ & $\begin{array}{c}\text { /Sedentário } \\
\mathbf{n}(\%)\end{array}$ & $\begin{array}{c}\text { Tabagismo } \\
\mathbf{n}(\%)\end{array}$ & $\begin{array}{c}\text { Alcoolismo } \\
\mathbf{n}(\%)\end{array}$ & $\begin{array}{c}\text { HAS } \\
\mathbf{n}(\%)\end{array}$ & $\begin{array}{c}\text { DM } \\
\mathbf{n}(\%)\end{array}$ \\
\hline $60-69$ anos & $35(67,3)$ & $23(41,8)$ & $36(64,3)^{*}$ & $4(7,1)$ & $9(16,1)$ & $32(57,1)$ & $8(14,3)$ \\
$70-79$ anos & $38(69,1)$ & $27(48,2)$ & $21(37,5)$ & $5(8,9)$ & $8(14,3)$ & $37(66,1)$ & $11(19,6)$ \\
$\geq 80$ anos & $12(46,2)$ & $7(26,9)$ & $8(32,0)$ & $5(19,2)$ & $2(7,7)$ & $20(76,9)$ & $2(7,7)$ \\
p-valor & 0,10 & 0,19 & 0,004 & 0,08 & 0,84 & 0,21 & 0,36 \\
\hline
\end{tabular}


...continuação - Tabela 2

\begin{tabular}{|c|c|c|c|c|c|c|c|}
\hline \multicolumn{8}{|l|}{ Sexo } \\
\hline Masculino & $22(64,7)$ & $7(20,6)$ & $12(35,3)$ & $2(5,9)$ & $10(29,4)^{*}$ & $18(52,9)$ & $4(11,8)$ \\
\hline Feminino & $63(63,0)$ & $50(48,1)^{*}$ & $53(51,5)$ & $12(11,5)^{*}$ & $9(8,7)$ & $72(68,6)$ & $17(16,2)$ \\
\hline $\mathrm{p}$-valor & 0,85 & 0,005 & 0,10 & 0,03 & 0,004 & 0,09 & 0,37 \\
\hline \multicolumn{8}{|l|}{$\begin{array}{l}\text { Arranjo } \\
\text { familiar }\end{array}$} \\
\hline Vive sozinho & $43(57,3)$ & $29(37,2)$ & $38(49,4)$ & $10(12,8)$ & $10(12,8)$ & $52(65,8)$ & $8(10,1)$ \\
\hline $\begin{array}{l}\text { Vive } \\
\text { acompanhado }\end{array}$ & $42(71,2)$ & $28(46,7)$ & $27(45,0)$ & $4(6,7)$ & $9(15,0)$ & $38(63,3)$ & $13(21,7)$ \\
\hline $\mathrm{p}$-valor & 0,09 & 0,26 & 0,61 & 0,47 & 0,18 & 0,76 & 0,06 \\
\hline \multicolumn{8}{|l|}{ Escolaridade } \\
\hline $\begin{array}{l}\text { Até o ensino } \\
\text { médio } \\
\text { incompleto }\end{array}$ & $81(64,8)$ & $53(41,1)$ & $58(45,3)$ & $12(9,3)$ & $17(13,2)$ & $84(64,3)$ & $21(16,2)$ \\
\hline $\begin{array}{l}\text { Ensino médio } \\
\text { complete } \\
\text { ou mais }\end{array}$ & $3(75,0)$ & $2(50)$ & $3(46,2)$ & $1(25,0)$ & $1(25,0)$ & $3(75,0)$ & $0(0)$ \\
\hline p-valor & 0,56 & 0,55 & 0,25 & 0,54 & 0,77 & 0,56 & 0,50 \\
\hline \multicolumn{8}{|l|}{$\begin{array}{l}\text { Classificação } \\
\text { econômica }\end{array}$} \\
\hline Classes A e B & $1(100)$ & $1(100)$ & $1(100)$ & $0(0)$ & $1(100)$ & $1(100)$ & $1(100)^{*}$ \\
\hline Classes C & $41(54,5)$ & $26(44,8)$ & $28(49,1)$ & $4(6,9)$ & $10(17,2)$ & $36(62,1)$ & $10(17,2)$ \\
\hline Classes D e E & $43(55,8)$ & $30(38,5)$ & $43(55,1)$ & $10(12,8)$ & $8(10,3)$ & $53(67,1)$ & $10(12,7)$ \\
\hline $\mathrm{p}$-valor & 0,06 & 0,37 & 0,50 & 0,68 & 0,10 & 0,63 & 0,04 \\
\hline \multicolumn{8}{|l|}{ Cor/Raça } \\
\hline Branca & $13(68,4)$ & $11(55,0)$ & $4(21,1)$ & $2(10,0)$ & $2(10,0)$ & $13(65,0)$ & $2(10,0)$ \\
\hline Não branca & $72(62,6)$ & $46(39,0)$ & $61(51,7)^{*}$ & $12(10,2)$ & $17(14,4)$ & $77(64,7)$ & $19(16,0)$ \\
\hline $\mathrm{p}$-valor & 0,62 & 0,18 & 0,013 & 0,87 & 0,79 & 0,59 & 0,38 \\
\hline
\end{tabular}

CCE: Circunferência abdominal elevada; IA: Insuficientemente ativo; HAS: hipertensão arterial sistêmica; DM: diabetes mellitus; ${ }^{*} p<0,05$.

\section{RESULTADOS}

O presente estudo identificou a prevalência de fatores de risco cardiovascular modificáveis e associou com as características sociodemográficas de idosos cadastrados em uma Unidade de Saúde da Família. Foi observada uma alta prevalência de idosos com hipertensão arterial $(64,7 \%)$, com sobrepeso/ obesidade $(63,1 \%)$, sedentarismo $(46,8 \%)$ e circunferência abdominal elevada (41,3\%).

Esses resultados foram semelhantes ao de 
um estudo realizado com idosos em Goiânia (GO), que também constatou que os fatores de risco cardiovascular mais prevalentes foram hipertensão $(80,4 \%)$, obesidade central $(76,2 \%)$, sedentarismo $(54,8 \%)$ e obesidade total $(27,0 \%)^{17}$.

No presente estudo, os achados mais relevantes foram às associações encontradas entre baixo nível de atividade física com grupo etário e raça, observando que $64,3 \%$ dos idosos entre 60 a 69 anos e 51,7\% dos não brancos encontraram-se insuficientemente ativos ou sedentários. Também foi observada associação do sexo feminino com circunferência abdominal elevada e tabagismo, mostrando que $48,1 \%$ e $11,5 \%$ das mulheres encontravam-se com circunferência abdominal elevada e fumantes, respectivamente. Enquanto o sexo masculino mostrou-se associado com alcoolismo, apontando que $29,4 \%$ dos homens faziam uso de álcool atualmente.

Outros autores têm apontado que dentre os fatores de riscos cardiovasculares, a HAS é o mais prevalente ${ }^{18}$ e aumenta com a idade $^{19}$. A maior prevalência de HAS no sexo feminino pode ser explicada porque as mulheres frequentam mais os serviços de saúde, podendo ser mais diagnosticadas com o problema em comparação aos homens ${ }^{20}$, visto que as informações de hipertensão utilizadas foram autorreferidas, baseadas em diagnóstico médico. Outra possível explicação para este achado é que os hormônios ovarianos são responsáveis por valores de pressão arterial mais baixos até o climatério, no entanto, após a menopausa, a carência desses hormônios tendem a elevar esses valores ${ }^{19}$.

Quanto à obesidade total, foi verificado uma menor prevalência com o avanço da idade, o que também foi evidenciado no estudo de Ferreira et al. ${ }^{17}$. Nenhuma associação significativa foi encontrada entre obesidade total e sexo, no entanto alguns estudos evidenciaram que a obesidade é maior entre as mulheres ${ }^{1,17,20}$.

Por outro lado, a obesidade central, verificada pela circunferência da abdominal elevada associou-se significativamente com o sexo feminino. Esta associação também foi evidenciada por outros estudos na literatura ${ }^{1,21}$. Segundo Wiltink et $\mathrm{al}^{22}$, a circunferência da cintura elevada apresenta-se como um fator de risco mais acentuado para as doenças cardiovasculares do que a obesidade geral, com maior prevalência nas mulheres ${ }^{23}$. Conforme apontaram Almeida R, Almeida M e Araújo ${ }^{24}$, o depósito de gordura na região abdominal das mulheres tem aumentado decorrente das mudanças socioeconômicas, que modificaram os hábitos alimentares e as condições de vida, expondo-as cada vez mais aos riscos cardiovasculares e à morbimortalidade por essas doenças.

Em relação ao sedentarismo, o presente estudo evidenciou uma associação com a cor/ raça, apontando osidosos não brancos combaixo nível de atividade física. Também foi observada uma associação entre sedentarismo e grupo etário, visto que os idosos de 60-69 anos foram mais sedentários que os idosos mais velhos. No estudo de Zaitune et al. ${ }^{25}$, os autores também observaram associação entre sedentarismo e idosos não brancos, concordando com os resultados da atual pesquisa, mesmo não sendo clara a relação existente entre cor/raça e a falta de atividade física. Porém, quando comparado sedentarismo e grupo etário, o estudo de Ferreira et al. ${ }^{17}$ constatou que a prevalência de sedentarismo aumentava nos idosos com idade mais avançada, sendo maior naqueles acima de 75 anos, discordando dos achados da atual pesquisa.

A associação verificada nesse estudo entre sedentarismo e grupo etário é bastante curiosa, pois o sedentarismo cresce conforme a idade avança ${ }^{26}$, o que não foi observado em nosso estudo e como a população idosa estudada apresenta baixo nível socioeconômico, esperava-se que os idosos mais jovens, ainda desempenhassem algumas atividades laborais, o que tenderia a aumentar o nível de atividade física.

Muitos resistem em praticar atividade física e muitas vezes essa resistência é provocada pelo desconhecimento sobre sua importância e benefício para o processo de envelhecimento ${ }^{27}$. Práticas de atividades física são fundamentais para a manutenção da saúde cardiovascular e em âmbito comunitário, a caminhada consiste no tipo de exercício mais conveniente e apropriado, visto que não exige o uso de equipamentos de alto custo, é simples, podendo ser realizada diariamente e apresenta 
baixo risco de complicações ${ }^{28}$.

Neste estudo, também foi observada associação entre tabagismo e sexo, apontando as mulheres com maior consumo de cigarro do que os homens. Esses resultados divergem de outros estudos que verificaram que o tabagismo é mais prevalente no sexo masculino e reduz nos idosos mais velhos ${ }^{10,29}$.

No presente estudo, acredita-se que a maior prevalência de mulheres fumantes reflita a elevada proporção de mulheres obesas, pois conforme verificado por Berto et al. ${ }^{10,}$ existe um grande número de mulheres obesas fumantes, o que pode ser justificado porque as mulheres obesas, possivelmente, fumam com o objetivo de perder peso.

Sabe-se que a idade e a obesidade são importantes fatores de risco cardiovascular e aliada à nicotina, que é altamente prejudicial à saúde, pode contribuir para o aumento da frequência cardíaca, da resistência periférica e consequentemente da pressão arterial, tendo seus efeitos sobre o sistema cardiovascular potencializado ainda por sua capacidade de formar coágulos e dificultar sua destruição ${ }^{28}$, intensificando o risco de adoecimento e morte.

A prevalência de alcoolismo entre os idosos pesquisados foi de 13,8\%, prevalência parecida foi verificada no estudo de Caetano et al. $^{28} \mathrm{em}$ idosos de Fortaleza (CE), e superior a encontrada por Ferreira et al. ${ }^{17}$, em idosos de Goiânia (GO) (6\%). O consumo de álcool foi associado ao sexo masculino, como também apontado em outros estudos ${ }^{17,20}$.

Com relação ao diabetes mellitus, observou-se uma proporção de $15,1 \%$ de idosos diabéticos, sendo que a maioria deles pertenceu ao sexo feminino e este foi o único fator de risco cardiovascular que foi associado à classificação econômica, sendo observado que o único idoso de classe A e B e 17,2\% dos de classe $C$ tinham diabetes, enquanto que e $12,7 \%$ daqueles de classe econômica D e E apresentaram a doença.

Moraes et al. ${ }^{30}$, ao investigar a prevalência de diabetes mellitus e fatores associados em adultos de Ribeirão Preto (SP), também verificaram que a situação econômica esteve associada ao diabetes, entretanto esses autores constataram que existe uma relação inversa de diabetes com a renda. Por outro lado, Mendes et al. ${ }^{31}$, ao avaliar a prevalência de diabetes mellitus e fatores associados em idosos em São Paulo (SP), observaram que não houve significância estatística entre condição econômica e diabetes autorreferida, apesar da prevalência de idosos diabéticos ser maior nos idosos de menor renda. Nesse sentido, a inconsistência observada entre os diferentes resultados na literatura, pode ser explicada pelos diferentes métodos utilizados para o diagnóstico de diabetes e/ou das variáveis econômicas e aponta para a necessidade de maiores investigações tentando elucidar essa associação.

Embora não tenha sido verificada nenhuma associação entre fatores de risco cardiovascular e arranjo familiar, é importante notar que viver sozinho pode comprometer a qualidade de vida dos idosos, especialmente naqueles que desenvolvem limitações funcionais, necessitando de auxílio de um cônjuge ou filho ${ }^{28}$.

As doenças cardiovasculares apresentam elevados impactos sobre o envelhecimento, assim o controle dos fatores de risco modificáveis é essencial para o planejamento de estratégias que contribuam para diminuir ou retardar o acometimento por essas doenças e garantir uma longevidade com qualidade de vida.

Destaca-se, que muitos desses fatores de riscos são influenciados por diferenças sociais, demográficas e econômicas, o que demanda uma atuação conjunta dos idosos e familiares, assumindo mudanças nos hábitos de vida; dos profissionais da saúde, sensibilizando a população para adoção de práticas saudáveis e gestores, viabilizando ações que garantam mudanças nos aspectos sociais e econômicos.

Os fatores de risco cardiovascular modificáveis devem ser abordados e priorizados durante a realização de estratégias de intervenção e prevenção das doenças cardiovasculares, com vistas à obtenção de mudanças nos hábitos de vida e garantia de um envelhecimento saudável e com qualidade.

Uma possível limitação deste estudo refere-se ao uso de medidas autorreferidas, para o diagnóstico de alguns fatores de risco cardiovascular, o que remete à possibilidade de ocorrência do viés de aferição. 


\section{CONCLUSÃo}

Neste estudo foi evidenciado uma elevada prevalência de fatores de risco cardiovasculares em idosos cadastrados em uma Unidade de Saúde da Família no município de Jequié (BA). A hipertensão arterial, o sobrepeso/ obesidade, o sedentarismo e a circunferência abdominal foram os fatores mais prevalentes, seguidos por diabetes, alcoolismo e tabagismo respectivamente.

Foi observada associação entre circunferência abdominal elevada e tabagismo com sexo feminino; sedentarismo com grupo etário mais jovem e com idosos não brancos; alcoolismo com sexo masculino e de diabetes mellitus com a classificação socioeconômica A e B.

\section{REFERÊNCIAS}

1. Carnelosso ML, Barbosa MA, Porto CC, Silva AS, Carvalho MM, Oliveira AL. Prevalência de fatores de risco para doenças cardiovasculares na região leste de Goiânia (GO). Cien Saude Colet. 2010; 15 Suppl 1:1073-80.

2. World Health Organization. Cardiovascular diseases (CVDs). [internet] 2015. [citado 2015 jul 10]. Disponível em: <http://www.who. int/mediacentre/factsheets/fs317/en/>.

3. Brasil, Ministério da Saúde. Indicadores de Saúde. [internet] 2015. [citado 2015 ago 13]. Disponível em: <http://tabnet.datasus.gov. br/cgi/tabcgi.exe?sim/cnv/obt10uf.def>.

4. Abranches LR. Inquéritos em Saúde no Brasil: relevância e fluxo informacional dos questionários [dissertação]. Rio de Jeneiro (RJ): Fundação Oswaldo Cruz; 2013.

5. Girotto E, Andrade SM, Cabrera MAS, Ridão EG. Prevalência de fatores de risco para doenças cardiovasculares em hipertensos cadastrados em unidade de saúde da família. Acta Scientiarum Health Sciences. 2009; 31(1):77-82.

6. Lessa I, Araújo MJ, Magalhães L, Almeida Filho N, Aquino E, Costa MCR. Simultaneidade de fatores de risco cardiovascular modificáveis na população adulta de Salvador (BA), Brasil. Rev Panam Salud Publica/Pan Am J Public Health. 2004; 16(2):131-7.

7. Brasil. Ministério da Saúde. Secretaria de Atenção à Saúde. Departamento de Atenção Básica. Prevenção clínica de doenças cardiovasculares, cerebrovasculares e renais - Brasilia: Ministério da Saúde, 2006.

8. Maia CO, Goldmeier S. Moraes MA, Boaz MR, Azzolin K. Fatores de risco modificáveis para doença arterial coronariana nos trabalhadores de enfermagem. Acta Paul Enferm. 2007; 20(2):138-42.

9. Muniz LC, Schneider BC, Silva ICM, Matijasevich A, Santos IS. Fatores de risco comportamentais acumulados para doenças cardiovasculares no sul do Brasil. Rev Saúde Pública. 2012; 46(3):534-42.

10. Berto SJP, Carvalhaes MABL, Moura EC. Tabagismo associado a outros fatores comportamentais de risco de doenças e agravos crônicos não transmissíveis. Cad Saúde Pública. 2010; 26(8):1573-82.

11. Brasil. Ministério da Saúde. Conselho Nacional de Saúde. Resolução no 466, de 12 de dezembro de 2012. Diário Oficial da União, Brasília (DF); 2013; [Citado 2015 jul 29]. Disponível em: http://conselho.saude.gov.br/resolucoes/2012/Reso466.pdf.

12. Craig CL, Marshall AL, Sjostrom M, Bauman AE, Booth ML, Ainsworth BE, et al. International Physical Activity Questionnaire: 12-country reliability and validity. Med Sci Sports Exerc. 2003; 35(8):1381-95.

13. BENEDETTI, Tânia Bertoldo; MAZO, Giovana Zarpellon; BARROS, Mauro Virgilio Gomes de. Aplicação do Questionário Internacional de Atividades Físicas para avaliação do nível de atividades físicas de mulheres idosas: validade concorrente e reprodutibilidade testereteste. Rev Bras Ciênc Mov. 2004; 12 (1): 25-34.

14. World Health Organization. Waist circumference and waist-hip ratio: report of a WHO expert consultation, Geneva, 8-11 December 2008 [Internet]. Geneva: World Health Organization;2008 [cited 2016 Fev 19]. Disponível em: http://apps.who.int/iris/ bitstream/10665/44583/1/9789241501491_eng.pdf

15. American Academy of Family Physicians, American Dietetic Association, National Council on the Aging. Nutrition screening e intervention resources for heal-thcare professionals working with older adults. Nutriftion Screening Initiative. Washington: American Diete_tic Association; 2002. [citado 2015 abr 07]. Disponível em: http://www.geriatria.unimore.it/wp-content/uploads/2016/01/ Nutrizione-nelle-patologie-croniche.pdf

16. Associação Brasileira de Empresas de Pesquisa. Critério de Classificação Econômica Brasil. 2010. [citado 2015 jul 29]. Disponível em: http://www.abep.org/criterio-brasil.

17. Ferreira CCC, Peixoto MRG, Barbosa MA, Silveira EA.Prevalência de Fatores de Risco Cardiovascular em Idosos Usuários do Sistema Único de Saúde de Goiânia. Arq Bras Cardiol. 2010, 95(5):621-8.

18. Sales JA, Tomaz CAB. Avaliação dos Fatores de Risco Cardiovascular e Alteração Eletrocardiográfica Pré-Operatória no Idoso Longevo. Rev Bras Cardiol. 2011; 24(1):9-17.

19. Silva JLL, Souza SL. Fatores de risco para hipertensão arterial sistêmica versus estilo de vida docente. Revista Eletrônica de Enfermagem. 2004; 06(03):330-335, 2004.

20. Pereira JC, Barreto SM, Passos VMA. O Perfil de Saúde Cardiovascular dos Idosos Brasileiros Precisa Melhorar: Estudo de Base Populacional. Arq Bras Cardiol. 2008; 91(1):1-10.

21. Nunes Filho JR, Debastiani D, Nunes AD, Peres KG. Prevalência de Fatores de Risco Cardiovascular em Adultos de Luzerna, Santa Catarina, 2006. Arq Bras Cardiol. 2007; 89(5):319-24.

22. Wiltink J, Michal M, Selvagem PS, Zwiener I, Blettner M, Münzel T, et al. Associations between depression and different measures 
of obesity (BMI, WC, WHtR, WHR). BMC Psychiatry, 2013.

23. Martins IS, Marinho SP. O potencial diagnóstico dos indicadores da obesidade centralizada. Rev Saúde Pública. 2003; 37(6):760-7. 24. Almeida RT, Almeida MMG, Araújo TM. Obesidade Abdominal e Risco Cardiovascular: Desempenho de Indicadores Antropométricos em Mulheres. Arq Bras Cardiol. 2009; 92(5):375-80.

25. Zaitune MPA, Barros MBA, César CLG, Carandina L, Goldbaum M. Fatores associados ao sedentarismo no lazer em idosos, Campinas, São Paulo, Brasil. Cad Saúde Pública. 2007; 23(6):1329-38.

26. Siqueira FV, Facchini LA, Piccini RX, Tomasi E, Thumé E. Silveira DS, et al. Atividade física em adultos e idosos residentes em áreas de abrangência de unidades básicas de saúde de municípios das regiões Sul e Nordeste do Brasil. Cad Saúde Pública. 2008; 24(1):3954.

27. Richter CM, Bettinelli LA, Pasqualotti A, Viecili PRN, Erdmann AL, Higashi GDC. Percepções de idosos sobre fatores de risco cardiovasculares. Rev Rene.2013; 14(4):996-1004.

28. Caetano JA, Costa AC, Santos ZMSA, Soares E. Descrição dos fatores de risco para alterações cardiovasculares em um grupo de idosos. Texto Contexto Enferm. 2008; 17(2):327-35.

29. Zaitune MPA, Barros MBA, Lima MG, César CLG, Carandina L, Goldibaum M. et al. Fatores associados ao tabagismo em idosos: Inquérito de Saúde no Estado de São Paulo (ISA-SP). Cad Saúde Pública. 2012; 28(3):583-95.

30. Moraes SA, Freitas ICM, Gimeno SGA, Mondini L. Prevalência de diabetes mellitus e identificação de fatores associados em adultos residentes em área urbana de Ribeirão Preto, São Paulo, Brasil, 2006: Projeto OBEDIARP. Cad Saúde Pública. 2010; 26(5):929-41.

31. Mendes TAB, Goldbaum M, Segri NJ, Barros MBA, Cesar CLG, Carandina L, et al. Diabetes mellitus: fatores associados à prevalência em idosos, medidas e práticas de controle e uso dos serviços de saúde em São Paulo, Brasil. Cad. Saúde Pública. 2011; 27(6):1233-43. 